\title{
A Case Report on Diabetes Mellitus Type 1 with Left Kidney Fungal Ball With Diabetic Nephropathy.
}

\author{
Dr. Bharat Veer Manchanda ${ }^{1}$,Dr. Girish Dubey ${ }^{2}$,Dr. Saloni Mehra ${ }^{3}$, \\ Dr. Umesh Verma ${ }^{4}$,Dr. Saminder Chaudhary ${ }^{5}$,Dr. Arun Khatri ${ }^{6}$ \\ ${ }^{1}$ Assi. Professor InDept Of Medicine, Subharti Medical College, Meerut \\ ${ }^{2,4,5,6}$ Post Graduate In Dept Of Medicine, Subharti Medical College, Meerut \\ ${ }^{3}$ Post Graduate In Dept Of Surgery, Subharti Medical College, Meerut
}

\begin{abstract}
In patient with Diabetes mellitus or impaired immunity lower UTI are common but it's a rare entity to see a renal fungal ball. Renal fungal ball or fungal bezor in the saprophytic colonization of a pre-formed cavity lay a conglomerate of fungal mycelia without invasion of adjacent tissue. Here we will be discussing a rare case of a young male 27 year old who was presented with T1 DM with left kidney fungal ball.
\end{abstract}

\section{Introduction}

Type $1 \mathrm{DM}$ is the result of interaction of genetic, environmental and immunological factors that ultimately lead to the destruction of the pancreatic beta cell and insulin deficiency. Type 1 DM which can develop at any age, developes most commonly before 20 year age. Worldwide the incidence of Type1 DM is increasing at the rate of 3-4\% per year for uncertain reasons. Individual with DM have a greater frequency and severity of infection. The reason include incompletely defined abnormalities in cell mediated immunity and phagocyte function associated with hyperglycemia as well as diminished vascularisation. Hyperglycemia aids the colonization and growth of variety of organism (Candida and other fungal species). The Incidence of Invasive fungal infection has risen dramatically in the last 20 years with the increased survival of patient with Immunocompromise states. These infection are often insidious and there diagnosis is usually delayed because of coexisting illness.

\section{Case Report}

A 27 year old male patient was admitted with complain of fever with chills \& Rigor, vomiting, generalized bodyache, pus in urine since 7-8 days. The patient had a history of T1DM since age of 9 years and was on Insulin (H.mixtard 30/70). Patient also underwent amputation of left leg below knee 4 year back and patient also gave history of diarrhea (on and off).

On Examination :- His general condition was unsatisfactory his initial blood pressure in left arm supine position was 130/90 and pulse rate of 88/min. Pallor was present, Ictreus, cyanosis, Edema, Lymphnode absent.Patient was conscious oriented to time, place, person CNS:-WNL

CVS :- S1S2+, No added sound, no murmur was heard

R/S:- B/L AE+, normal vesicular breath sound,

P/A:- Soft, Non-tender, No-palpable organomegaly

His initial investigation revealed microcytic, hyprochromic anemia with lucocytosis (Hb 7.2 method photometry, Wbc 25.2 method electrical impedence), Derranged RFT (B.urea 441, method urease with Indicator dye, S.cret 9.50 method enzymatic, Na114 method Direct ISE, K 4.40 method direct ISE) LFT WNL.

Urine routine/Microscopy revealed pulse as 40-45/HPF, RBC 8-9/HPF, Epithelial Cell 1-2/HPL, Albumin +, Sugar+, Pseudo Hyphae, Budding yeast+ method Dipstic Reflectance Spectrophotometry/Microscopy. Viral markers were negative.

USG (W/A) was done which was suggestive of

Right Kidney $-8.6 \times 3.6 \mathrm{~cm}$ altered echotexture

Left Kidney - Grossly hydronephrotic. CMD totally lost with various multiple size calculus, largest measuring $1.57 \mathrm{~cm}$.

Imp:- PUJ obstruction with multiple calculi with gross hydronephrosis.

A urosurgery opinion was taken in view of PUJ obstruction with multiple calculi with gross hydronephrosis and NCCT KUB was advised.

NCCT KUB Revealed:- Left Kidney grossly dilated 
Pevicalyceal system noted with thinking of Cortex. Multiple round hyperdensities measuring $1 \times 1 \mathrm{~cm}$ noted within the dilated pelvicalyceal system of left kidney? Fungal ball.

Right kidney:- Normal in size shape position CMD maintained

CECT was not done due to increased renal parameters.

Urine $\mathrm{KOH}$ was done which came out to be positive for fungal element.

Treatment:-

Cap.itraconazole $100 \mathrm{mg} \mathrm{BD}$

\section{Discussion}

Individual with DM have a greater frequency and severity of infection. The reason for this include incompletely defined abnormalities is cell - mediated immunity and phagocyte function associated with hyperglycemia, as well as diminished vascularisation. Hyperglycemia aids the Colonization and growth of a variety of organisms Candida and other fungal species. Many common infection are more frequent and severe is diabetic population, whereas several rare infections are seen almost exclusively in the diabetic population.

Upper urinary fungal tract infection are relatively uncommon and fungal ball (before) formation in particularly rare entity.Renal involvement has been reported $8-13 \%$ of patients with disseminated disease. Most $80 \%$ have unilateral involvement.

The incidence of invasive fungal infections has risen dramatically in the last 20 years with increased survival of patients with immunocompromised state.These infections are often insidious and their diagnosis is usually delayed because of coexisting illness. There are many fungi which may involve deeper tissues including kidney. Candida \& Aspergillus species are common cause but zygomycetes, Cryptococcus and dimorphic fungi (e.g. Histoplasma, coccidioides, Blastomyces and Penicillicem) Species may also rarely involve kidney.Most invasive fungal infections are caused by hematogenous spread.Animal experiments have shown that fungi injected intravenously are usually cleared from bloodstream. In the immunocompromised host, however, blood borne fungi infect the kidney's by attachement to endothelial surface followed by penetration into the interstitium and multiplication.Ascending infection of the kidney may also be important in a minority of individuals. Risk factor for the multiplication of the organisms in renal tissue include; AIDS, diabetes, lymphoma, leukemia, burns, Renal failure, organ transplantation and immunosuppressive drugs.Renal fungal infection may present in following ways. Disseminated fungal infection with Renal involvement. It results from hematogenous spread of fungi to the kidney leading to formation of multiple focal abscess.Fungal infection of Renal Pelvis. It results in obstructive uropathy which may present with urinary retention. Ascending panurothelial fungal infective. This refers to infection involving urethera, bladder, ureter and kidney.Majority of the cases described in the literature suggest and diabeties, Immunosupressed state, bladder Catherization and prolonged antibiotic use are a few high risk factor for opportunistic infection by fungus.Literature review reveals that disseminated fungal infection can involve renal system commonly but primary Ascending infection can also involve renal parenchyma, can cause PUJ obstruction and present as pyuria, Fever with chills \& Rigor, hematuria, oliguria and Anuria.

\section{Refrences}

1. Alvin C. Powers "Diabetes mellitus:Diagnosis,classification and pathophysiology". Harrison's Principle of internal medicine, Kasper, Fauci, Hauser, Longo, Jameson et al: Mc graw hill education vol2 ,edition 192015 page no.2136 ISBN978-0-07180214-7.

2. K.L gupta: "Fungal infections and the kidney". Oxford desk refrences nephrology. Jonathan Baratt, Kevin Harris , Peter Topham. Oxford university press 2009 . ISBN 978-0-19-922956-7.

3. Devimeenal jegannathan, Kanagasbai Ramanathan :Renal fungal ball-two case reports and review of literature. Doi :http://dx.doi.org/10.1259/bjcr:20150247 